\title{
« La flamme dévoratrice d'offrandes »: feu et agentivité rituelle dans la tragédie grecque
}

"The Offering-Eating Flame": Fire and Ritual Agency in Ancient Greek Tragedy

\section{Gloria Mugelli}

\section{(2) OpenEdition}

\section{Journals}

Édition électronique

URL : http://journals.openedition.org/mondesanciens/2406

DOI : 10.4000/mondesanciens. 2406

ISSN : 2107-0199

\section{Éditeur}

UMR 8210 Anthropologie et Histoire des Mondes Antiques

\section{Référence électronique}

Gloria Mugelli, « « La flamme dévoratrice d'offrandes » : feu et agentivité rituelle dans la tragédie grecque », Cahiers «Mondes anciens » [En ligne], 12 | 2019, mis en ligne le 01 avril 2019, consulté le 01 mai 2019. URL : http://journals.openedition.org/mondesanciens/2406 ; DOI : 10.4000/ mondesanciens. 2406

Ce document a été généré automatiquement le 1 mai 2019.

Les Cahiers «Mondes Anciens » sont mis à disposition selon les termes de la licence Creative Commons Attribution - Pas d'Utilisation Commerciale - Pas de Modification 4.0 International. 


\title{
« La flamme dévoratrice
} d'offrandes » : feu et agentivité
rituelle dans la tragédie grecque

"The Offering-Eating Flame": Fire and Ritual Agency in Ancient Greek Tragedy

\author{
Gloria Mugelli
}

1 Le verbe le plus générique pour indiquer un sacrifice (sanglant ou non), $\theta u ́ \omega$, désigne plus précisément l'offrande par combustion et la production de fumée ${ }^{1}$. La présence de feu sur l'autel est en effet un des éléments constants dans toute la démarche sacrificielle grecque, à partir des phases préparatoires du rite jusqu'aux dernières libations de vin, qui éteignent la flamme. Élément indispensable pour le rite, le feu est le principal moyen de consécration des offrandes alimentaires ${ }^{2}$ et il est actif dans tous les processus qui déterminent l'efficacité des sacrifices sanglants: la combustion des portions de viande destinées aux dieux, la cuisson des portions réservées aux hommes et la production de la кvíon, la fumée grasse qui monte jusqu'aux dieux ${ }^{3}$. Les textes tragiques mentionnent plusieurs sacrifices et offrandes, dont la plupart n'était pas représentée sur scène : ils étaient censés se dérouler dans l'espace extra-scénique invisible, et étaient donc décrits ou mentionnés par les personnages ou par le chœur tragique.

2 Les études à propos des rituels, et notamment des sacrifices, dans la tragédie grecque ont mis l'accent sur le mécanisme dramatique du "perverted sacrifice», dans lequel les meurtres sont décrits sous le registre métaphorique de l'égorgement sacrificiel $(\sigma \varphi \alpha \gamma \eta ́){ }^{4}$. Néanmoins, les représentations des rites dans la tragédie grecque ne se limitent pas au pattern du rite corrompu et, dans plusieurs passages tragiques, la description des différents aspects du rite a une fonction dramatique bien précise dans le déroulement des événements du drame. Les rites représentés ou décrits sur la scène tragique peuvent être considérés en tant que ressources dramaturgiques, employées par les auteurs tragiques dans la construction de l'intrigue du drame ${ }^{5}$.

3 Dans la construction d'images rituelles plus ou moins élaborées, les dramaturges pouvaient compter sur les compétences et les expériences rituelles partagées par le 
public: en tant que citoyens d'Athènes, les spectateurs prenaient part à toutes les

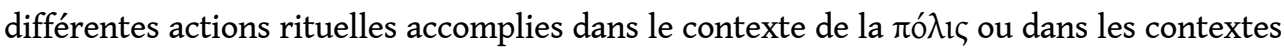
familiaux. Certains rites étaient donc une expérience récente (voire concomitante) pour les participants aux Grandes Dionysies, et d'autre part, la participation en tant que spectateurs à la performance rituelle de la tragédie était en elle-même une forme d'activité rituelle ${ }^{6}$. Ce travail porte sur le croisement entre expérience du rite et expérience de la tragédie, en analysant les différentes modalités de mise en image, dans les textes tragiques, d'un élément central de la pratique sacrificielle : l'image du feu qui brûle sur les autels est souvent évoquée par les poètes tragiques pour indiquer la présence d'une activité rituelle, d'autant que l'image des autels sans feu implique l'absence de sacrifices ou leur inefficacité.

La métaphore du feu "vif », employée en français aussi bien que dans d'autres langues modernes, met en évidence la possibilité de penser le feu en tant qu'«objet vivant »: élément actif et mobile sans doute, le feu se montre évidemment pourvu de quelques marges d'actions quand il échappe au contrôle des hommes.

Les modes d'action des «choses" dans la démarche sacrificielle ne se limitent toutefois pas à la substitution de l'action des hommes ${ }^{7}$, où à la reproduction des processus de l'agentivité humaine ${ }^{8}$ : lors du sacrifice, comme lors de toute action rituelle, les objets, les "choses ", font partie du réseau d'actions qui constituent un rite efficace ${ }^{9}$. Il faut donc s'interroger sur les formes de la présence du feu sacrificiel dans les différentes phases du rite à partir de son rapport aux corps des agents rituels en action dans les espaces du sacrifice ${ }^{10}$. L'objectif de notre réflexion n'est donc pas de considérer les différents dégrés d'agentivité ou personnification du feu en tant qu'objet: il s'agit plutôt d'explorer les interactions entre le feu, ou en général les « choses » présentes au cours de la démarche sacrificielle, et les agents humains et divins du sacrifice. Je me propose donc de discuter les attestations tragiques de l'image du feu sur les autels, en mettant en relief les différents modes d'actions de cet élément. À la fois produit de l'initiative des sacrifiants et indice du déroulement correct $\mathrm{du}$ rite, l'image $\mathrm{du}$ feu peut évoquer, d'une part les différents modes d'action des hommes au sacrifice, et d'autre part, elle peut indiquer la présence et la faveur des dieux. En tant qu'élément sur lequel se concentrent les actions des hommes et des dieux lors d'un sacrifice, le feu peut mettre en image les différentes dynamiques de l'efficacité des rites.

En premier lieu, j'aborderai la question de la préparation et de l'allumage du feu du sacrifice, en rapport avec les agents qui accomplissent le rite. En deuxième lieu, j'analyserai l'action du feu dans la production des signes de l'efficacité du rite (la beauté de la flamme et ses effets sur les offrandes), qui nous permettront de mettre en question les différents modes d'action divine à l'occasion du sacrifice. Enfin, je mettrai en rapport les images de la flamme du sacrifice avec une image mythique, le bûcher du suppliant, qui représente une forme d'action différente du feu sur les autels, et témoigne d'une action partagée entre les hommes et les dieux lors de rites de supplication.

\section{Allumer le feu : les agents du sacrifice}

7 Le sacrifice d'Iphigénie est sans doute le rite le plus présent dans le corpus des tragédies conservées jusqu'à nos jours. À la fin de l'Iphigénie à Aulis d'Euripide, Iphigénie met en marche la procession qui l'escorte à l'autel d'Artémis, et commande que quelqu'un allume le feu sacrificiel ${ }^{11}$. L'approvisionnement en bois et en charbon était un des premiers 
soucis des sacrifiants, à l'occasion des grands sacrifices publics ou des holocaustes, qui demandaient une très grande quantité de combustibles pour consumer entièrement les victimes.

Plusieurs lois sacrées spécifient la quantité de bois à acheter pour les sacrifices: l'inscription $d u \mathrm{IV}^{\mathrm{e}}$ siècle du dème d'Aixônè, en Attique, règlemente la fourniture des $\varphi p u ́ \gamma \alpha v \alpha$, le bois pour allumer le feu, et des $\xi u ́ \lambda \alpha$, le bois pour brûler les offrandes, à l'occasion des différentes pratiques sacrificielles ${ }^{12}$. Le үévoৎ attique des Salaminioi dépensait 530 drachmes et 3 oboles chaque année pour le bois des sacrifices ${ }^{13}$. Selon une inscription du milieu du IV siècle, le prêtre d'Asclépios à Épidaure ${ }^{14}$ demandait un demiobole aux sacrifiants qui étaient dépourvus de bois à leur arrivée dans le sanctuaire. Le feu est également un des éléments nécessaires pour démarrer le rite dans la démarche des sacrifices familiaux : lors du sacrifice aux Nymphes, célébré hors scène dans l'Électre d'Euripide, les esclaves d'Égisthe allument le feu et préparent les instruments (sphageion, lebes et corbeille) pour accomplir le rite ${ }^{15}$. La préparation des instruments et des matériaux pour le sacrifice, très rarement évoquée dans la tragédie, est un expédient dramatique employé plutôt fréquemment sur scène dans la comédie, ce qui témoigne de l'importance de cette phase du sacrifice ${ }^{16}$. Aristophane représente sur scène plusieurs sacrifiants en train de se rendre au sacrifice avec les matériaux pour allumer le feu : dans les Thesmophories (v. 36-38), les charbons sont transportés dans un brasier portatif, alors que dans quelques passages tragiques, l'action d'allumer un feu indique l'initiative des sacrifiants qui, si le sacrifice est empêché par des conditions défavorables, renoncent à allumer la flamme ${ }^{17}$. L'action de l'allumage définit également les intentions des agents du rite : dès qu'une bonne nouvelle arrive aux citoyens ${ }^{18}$, leur réaction joyeuse et festive est

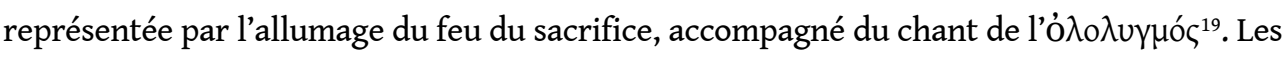

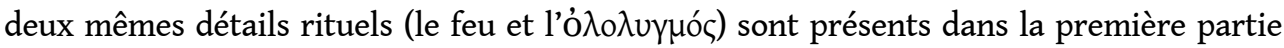
de l'Agamemnon d'Eschyle, où la description d'un sacrifice met en rapport l'image du feu qui brûle les offrandes avec les différents agents du rite. L'image de la flamme est évoquée au début de la tragédie, quand les messages des flambeaux annoncent la prise de Troie et le retour du héros.

9 Avant l'entrée en scène du héraut, qui les confirme, l'action dramatique est focalisée sur la réception du message par Clytemnestre et le chœur. La reine fait confiance à l'annonce de la victoire et donne l'ordre de sacrifier en raison de la bonne nouvelle ${ }^{20}$. Ces sacrifices posent les conditions de la performance dramatique : le chœur arrive dans l'orchestra, en face du palais d'Agamemnon, pour demander à Clytemnestre la raison des sacrifices, et décrit, dans la parodos, les rites qui sont en train de se dérouler hors scène : tous les autels

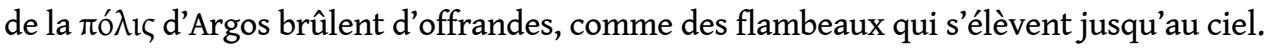

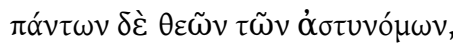

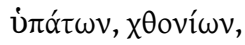

$\tau \tilde{\omega} v \tau^{\prime}$ o ${ }^{\prime} \rho \alpha v i \omega v \tau \tilde{\omega} v \tau^{\prime} \dot{\alpha} \gamma o \rho \alpha i ́ \omega v$,

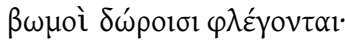

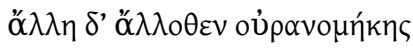

$\lambda \alpha \mu \pi \grave{\alpha} \varsigma \dot{\alpha} v i ́ \sigma x \varepsilon l$,

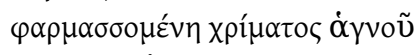

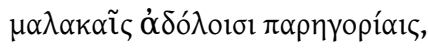

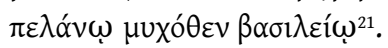

Les autels de tous les dieux qui tiennent la ville, ceux d'en haut, ceux de la terre, ceux du ciel et ceux de l'agora, brûlent d'offrandes. D'un côté et de l'autre, la 
flamme se dresse jusqu'au ciel, charmée par la persuasion douce et sincère d'un onguent sacré, le pelanos royal tiré du cœur du palais.

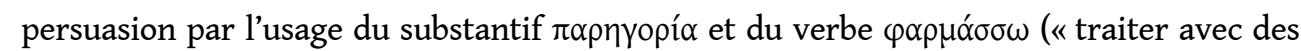
poisons/médicaments »)22. L'adjectif ơ $\delta \circ \lambda$ os est également significatif: la sincérité des intentions rituelles de Clytemnestre est mise en question dans l'intrigue de la tragédie. La métaphore qui compare les autels aux flambeaux fait le lien entre le sacrifice et les messages lumineux : l'image évoquée est celle d'une course aux flambeaux métaphorique (au vers 312 les ordres de Clytemnestre pour les messagers sont décrits comme les règles

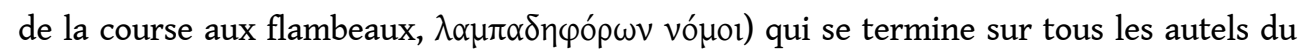
sacrifice.

11 moment de l'allumage du feu était souvent ritualisé. Lors des Panathénées, par exemple, la $\lambda \alpha \mu \pi \alpha \delta \eta \delta \rho \mu^{\prime} \alpha$, la course aux flambeaux, parcourait le même itinéraire que la procession, et se terminait sur l'autel du sacrifice ${ }^{23}$. La plupart des attestations iconographiques de la course aux flambeaux clarifie le rapport entre les flambeaux et l'autel : le flambeau des vainqueurs allumait le feu sur l'autel. Clytemnestre participe aux rites dans la cité d'Argos en premier lieu avec l'envoi du personnel chargé des sacrifices, les Өvoбkहĩ (v. 87). Le rôle de la reine a toutefois aussi des conséquences sur les effets du sacrifice : la flamme sur l'autel est "charmée" par un $\pi \varepsilon \lambda \alpha v o ́ \varsigma$ qui vient de la maison du roi (v. 94-97). Attesté à Delphes et à Éleusis en tant que taxe sacrificielle ${ }^{24}$, le $\pi \varepsilon \lambda \alpha v o ́ \varsigma$ (une bouillie plus ou moins liquide composée de miel, huile et farine qui pouvait être versée dans des libations ou bien pétrie dans des gâteaux) est l'offrande non sanglante tragique par excellence ${ }^{25}$, souvent associée au feu sacrificiel. La bonne combustion du $\pi \varepsilon \lambda \alpha$ ó beauté du feu sacrificiel sont souvent liées à l'efficacité du rite. Dans l'Ion d'Euripide (v. 705-708) le $\pi \varepsilon \lambda \alpha v o ́ c$ est - selon toute probabilité - mentionné dans le contexte d'un sacrifice sanglant accompli à Delphes: le chœur vient d'entendre la proposition de Xouthos d'adopter Ion par la célébration des $\gamma \varepsilon v \varepsilon \varepsilon \lambda_{l} \alpha$, accompagné du sacrifice d'un bœuf

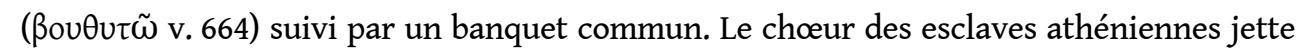
une malédiction sur Xouthos, qui vise les résultats et les effets du sacrifice: elles souhaitent que le $\pi \varepsilon \lambda \alpha v o ́ \varsigma$ sacrifié par Xouthos ne donne pas une belle flamme ${ }^{26}$. Dans le passage de l'Agamemnon mentionné précédemment, la hauteur de la flamme sacrificielle signale donc le déroulement correct du rite. La provenance de l'offrande de l'intérieur de la maison du roi connecte le rite à son agent. H. Hubert et M. Mauss (1897) distinguaient le rôle du sacrifiant (celui qui avait l'intention de sacrifier et payait pour le rite) et le rôle du sacrificateur (celui qui accomplissait l'action sacrée, ou y participait). R. Parker (2009) a, toutefois, démontré que cette distinction ne s'applique pas au sacrifice grec, dès lors que l'action de Gúciv est accomplie sans distinction par ceux qui prennent la décision de sacrifier, ceux qui payent pour les victimes et les matériaux, ceux qui accomplissent l'action rituelle, mais aussi ceux qui assistent au rite et participent au banquet.

La représentation de Clytemnestre en tant qu'agente du sacrifice est d'autant plus efficace, dans la dynamique de la première partie de la tragédie, dans la mesure où le chœur met en question la sincérité de Clytemnestre dans sa joie pour le retour du mari : le $\pi \varepsilon \lambda \alpha v o ́ \varsigma$ de la reine qui «enchante la flamme » détermine l'efficacité d'un sacrifice spectaculaire. 
13 Malgré la méfiance du chœur, dans le deuxième épisode, le héraut confirme la validité de l'annonce du retour d'Agamemnon. Clytemnestre affirme que, à la différence du chœur, elle n'a pas eu besoin d'attendre la validation des messages lumineux pour accomplir le

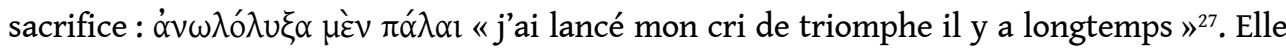
décrit les sacrifices en évoquant la présence de toute la communauté d'Argos qui fait confiance à sa reine et participe au sacrifice. La participation au rite est mise en image avec une action que les sacrifiants accomplissent sur le feu du sacrifice: les citoyens

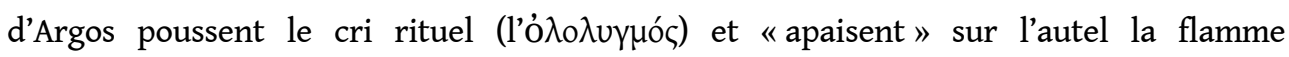
"dévoratrice d'offrandes $»^{28}$. Dans l'Agamemnon, les différentes descriptions de la flamme sacrificielle qui brûle sur les autels mettent en image, d'une part, l'attitude de Clytemnestre et son initiative rituelle et, d'autre part, la participation de la cité d'Argos au sacrifice. Ces images, qui se fondent sur l'expérience rituelle du public de la tragédie, sont d'autant plus efficaces dans la construction des événements du drame si on considère que, dès le début de la tragédie, le public connaît les véritables intentions de Clytemnestre.

L'Agamemnon représente donc un sacrifice hypocrite mais apparemment efficace: la flamme qui se lève sur l'autel et dévore les offrandes est un des signes du déroulement correct du rite.

\section{Les signes de la flamme à l'autel : bons et mauvais sacrifices}

Dans la célèbre rhesis du Prométhée enchainé, Prométhée détaille les différentes techniques de divination sacrificielle qu'il a apprises aux mortels. Une partie des signes provient des entrailles (la couleur et le poli des viscères, la forme du lobe du foie); l'autre est à lire

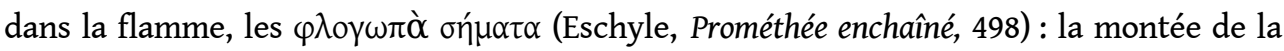
queue sur l'autel, la combustion des cuisses enveloppées par la graisse, la coloration de la flamme par la bile.

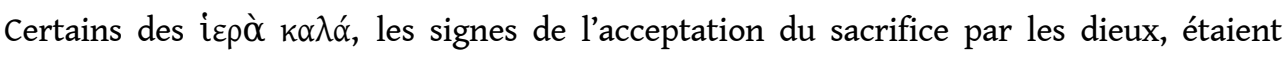
produits par des réactions causées par le feu. Ainsi, M.Jameson et G. Ekroth ont

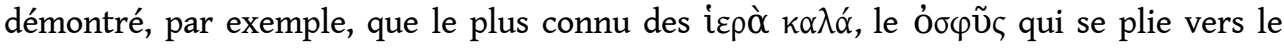
haut, n'est rien d'autre qu'une réaction de la queue du bœuf à la chaleur du feu, et que de

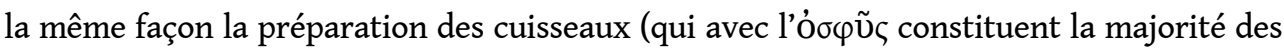
trouvailles zoo-archéologiques dans les sanctuaires) avec l'os enveloppé par le gras, avait comme effet - dès lors qu'on place la cuisse sur le feu - la montée de la flamme du sacrifice ${ }^{29}$. Enfin, la vésicule biliaire était pressée sur le feu, de telle façon que la bile puisse colorer la flamme ${ }^{30}$. Les signes issus du sacrifice présentaient différents niveaux de

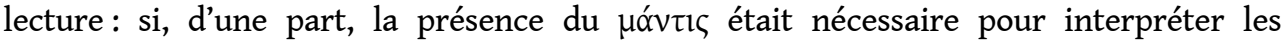
présages, d'autre part, les sacrifiants pouvaient reconnaitre les signes les plus évidents ${ }^{31}$. Lors du sacrifice accompli sur scène par Trygée et l'esclave dans la Paix d'Aristophane, au moment de la combustion de la part des dieux, les deux sacrifiants s'exclament que «la queue se plie » selon la norme (v. 1055). Dans ce passage, le comique était probablement engendré par les détails de la mise en scène, qui nous échappent, et par les gestes qui accompagnaient les mots. Tout de même, ce passage nous permet de comprendre que le signe de la queue était beau à voir et facile à reconnaitre aussi pour les sacrifiants les plus inexpérimentés. 
17 D'une certaine façon, donc, la présence d'un feu suffisamment vif et la compétence rituelle nécessaire pour préparer les parts de viande étaient la garantie de la bonne réussite du sacrifice, qui produisait le plaisir rituel pour tous les participants : le sacrifice était beau à voir, mais aussi bon à sentir et à manger.

Le feu sur l'autel est le signe de la faveur des dieux, de leur volonté d'accepter le sacrifice ou tout simplement de leur présence dans l'espace sacré.

Dans les Troyennes d'Euripide, la représentation de Troie détruite par les Achéens passe par la description d'une ville sans rite, où il n'y a pas de place pour les danses, les chœurs, le feu sacrificiel: dans le prologue, par exemple, Poséidon décrit les lieux sacrés abandonnés et les temples de la ville couverts de sang, notamment le temple de Zeus herkeios où Priam a été tué (Euripide, Troyennes, 15-17). Poséidon lui-même va abandonner ses autels à Troie parce que dans une ville déserte il n'y a pas de place pour le culte des

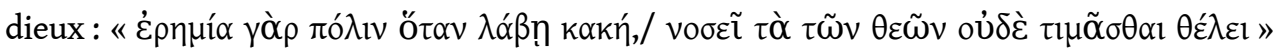
(Euripide, Troyennes, 26-27).

20 En même temps, si, d'une part, la guerre et l'impiété des Grecs sont responsables de l'interruption de l'activité rituelle et de la violation des temples par la mise à mort des suppliants qui causent l'abandon des autels par Poséidon, d'autre part, la destruction de Troie est attribuée à l'absence des dieux : le chœur des Troyennes se plaint que les dieux aient abandonné leur ville. Zeus en particulier a confié aux Achéens son temple et son autel qui était parfumé par les offrandes des $\pi \varepsilon \lambda \alpha v o i ́$ et par la fumée de la myrrhe (v. 1063-1070).

21 L'absence du feu signifie donc non seulement l'absence des sacrifiants, et de bonnes raisons pour sacrifier, mais elle signifie également l'absence des dieux, ou leur refus de consommer le sacrifice : les $\pi \varepsilon \lambda \alpha v o i ́$ ne sont pas touchés par la flamme (ö $\varphi \lambda \varepsilon \kappa \tau o l)$, quand la colère de Déméter rend la terre stérile et interrompt toute activité rituelle (Euripide, Hélène, 1333-1334).

22 Un passage très connu d'Antigone constitue une des rares représentations d'un sacrifice inefficace, où la viande sacrificielle n'est pas consommée par le feu. Une fois qu'il a obtenu des présages défavorables par les oiseaux, Tirésias essaye de lire les ع̌ $\mu \pi v \rho \alpha$, les signes qui viennent des sacrifices par le feu. L'issue du rite est épouvantable : la flamme ne brûle pas les offrandes, de sorte que les cuisseaux et la graisse se décomposent sur l'autel, tandis que la vésicule biliaire s'écrase (v. 1005-1022).

23 Le sacrifice se caractérise par l'absence des signes issus du feu qui traduisent la réussite $\mathrm{du}$ rite: quand il apprend les résultats du sacrifice, Tirésias ne parle pas de présages défavorables, mais plutôt d'ópүı ǒ $\sigma \eta \mu \alpha$, de « rites sans signes ». Les marges de l'action (et du manque d'action) divine dans ce sacrifice sont strictement liées aux effets du feu : les signes de la mauvaise réussite du rite sont attribués à Héphaïstos lui-même, qui ne brûle pas les offrandes sur l'autel.

24 De la mauvaise réussite du sacrifice, Tirésias peut deviner que les dieux sont en colère, et n'acceptent ni les prières, ni la flamme des cuisses offertes en sacrifice. La raison de cette colère, et de la contamination de la cité de Thèbes est bien significative : les oiseaux ont transporté la chair du cadavre de Polynice sur tous les autels de la ville, et les dieux ont donc bu de la graisse et du sang humains.

Les oiseaux sont les perturbateurs par excellence du sacrifice : les oiseaux d'olympie, qui ne se jettent pas sur la viande sacrificielle, sont vus comme une exception remarquable, 
qui témoigne de la sacralité du temple de Zeus. Normalement, dit Pausanias, un oiseau qui vient picorer les entrailles ou la viande sacrificielle est un très mauvais présage ${ }^{32}$. Dans l' Antigone, les oiseaux déposent sur l'autel de la viande qui n'est pas censée être consommée par les dieux, et, faisant couler le sang sur l'autel, ils interrompent le déroulement ordinaire des pratiques sacrificielles. En même temps, en l'absence de feu, la viande sacrificielle offerte en sacrifice par Tirésias subit le même sort que la chair du cadavre laissé sans sépulture : elle se décompose et pourrit.

Les passages que nous avons analysés représentent différents modes d'interruption de la communication sacrificielle entre hommes et dieux.

27 À Troie les dieux ont abandonné la ró $\lambda_{1 \varsigma}$ à la destruction et à l'impiété des Grecs, qui trouve son summum dans la mise à mort de Priam sur l'autel de Zeus : toute action sacrée devient par conséquent impossible. À Thèbes les humains ont violé les lois des dieux en laissant le cadavre de Polynice sans sépulture et, avec la complicité de l'action des oiseaux, ont déposé sur les autels de la viande humaine qui n'est pas bonne à manger pour les dieux.

Cela témoigne de l'impossibilité de la coexistence du feu et du sang humain sur les autels : si, d'une part, la contamination de l'espace sacré cause l'absence des dieux, et donc l'absence de feu et d'activité rituelle, d'autre part, la présence sur l'autel de viande sacrificielle non consommée par le feu, qui se décompose, est un signe évident de la contamination.

\section{Le bûcher du suppliant, feu sur les autels et agentivité divine}

Avant de tirer quelques conclusions, le dossier des actions mobilisées par la présence du feu sur les autels peut être enrichi avec un dernier exemple qui n'est pas en lien avec le sacrifice tragique, mais avec la supplication. Ce rite est représenté dans plusieurs tragédies, et il permet aux auteurs tragiques de mettre en scène une situation de tension, où l'espace sacré est menacé par la possibilité que le suppliant soit mis à mort sur l'autel. Même si, dans les tragédies conservées, les suppliants ne meurent jamais dans l'espace sacré représenté sur scène, les confrontations entre suppliants et suppliés présentent aux spectateurs les possibles résultats violents du rite.

Une fois qu'un suppliant était installé sur un autel, le rite de la supplication pouvait avoir une bonne ou une mauvaise issue. Si la supplication était inefficace, le supplié ou ceux qui avaient l'autorité sur l'espace sacré pouvaient simplement refuser la requête du suppliant. Si la situation l'exigeait, ils pouvaient mettre fin au rite d'une façon violente, en expulsant le suppliant du lieu sacré ou en le mettant à mort ${ }^{33}$ : la mise à mort de Priam, égorgé sur l'autel de Zeus, constitue le paradigme de l'impiété envers les suppliants, et dans les Troyennes elle est représentée en tant qu'alternative au déroulement régulier des pratiques sacrificielles. Dans la tragédie, l'interférence entre échec de la supplication et $\sigma \varphi \alpha \gamma \eta ́$ sacrificielle est souvent évoquée par les suppliants qui défient leurs agresseurs de faire couler le sang dans l'espace sacré. Les agresseurs, pour leur part, envisagent plutôt des stratégies de mise à mort "non sanglante »: la précipitation, la lapidation et le bûcher. Dans deux tragédies d'Euripide, Andromaque ${ }^{34}$ et Héraclès, cette dernière forme de mise à mort est présentée comme une façon alternative de tuer les suppliants par rapport à l'égorgement sur l'autel. Dans l'Héraclès, le bûcher sur l'autel est sur le point d'être mis 
en place sur scène: Lycos donne l'ordre d'aller chercher le bois pour mettre le feu à l'autel de Zeus $\Sigma \omega \tau$ tn où Mégara, Amphitryon et les enfants d'Héraclès se sont installés en suppliants au début de la tragédie (v. 243-246). Menacés de mort par le feu, les suppliants décident de quitter l'autel et se livrent à Lycos en choisissant la mort par le couteau ( $\sigma \varphi \alpha \gamma \eta ́)$, plus honorable que la mort à l'autel ${ }^{35}$. Dans l'interprétation de Naiden, le bûcher est "une forme accélérée de barricade ${ }^{36}$ »: il n'a pas pour but de tuer le suppliant à l'intérieur du sanctuaire, mais plutôt de l'éloigner de l'espace sacré. Hors de la tragédie, toutefois, nous avons plusieurs attestations de supplications qui aboutissent à la mort du suppliant par le feu, qui peuvent éclairer le rôle du feu dans les dynamiques de la supplication. Si, d'une part, l'agresseur des suppliants qui met le feu à l'espace sacré peut être puni pour son impiété37, d'autre part le dispositif du bûcher sollicite, dans quelques cas, l'intervention des dieux, qui expriment leur jugement en choisissant de mettre à mort le suppliant ${ }^{38}$ ou d'éteindre le feu: dans le premier livre des Histoires d'Hérodote (I, 86), Cyrus met ainsi à mort Crésus par le feu avec l'intention de voir si un dieu l'aurait

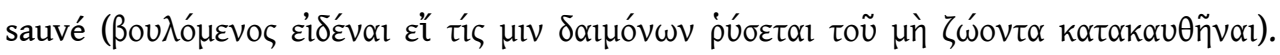
Apollon envoie en effet un orage pour éteindre le feu. Cette tension entre responsabilité humaine ${ }^{39}$ et jugement des dieux dans la mise en place du bûcher des suppliants est bien évidente dans l'épisode de la mise à mort d'Alcmène, qui fonctionne très probablement de contrepoint mythique à l'évocation du bûcher dans l'Héraclès d'Euripide.

Dans ce récit mythique, mis en image dans des représentations iconographiques qui pourraient être inspirées de l'Alcmène d'Euripide ${ }^{40}$, après avoir découvert l'adultère, Amphitryon aurait mis à mort sa femme sur un bûcher. Zeus intervient donc en envoyant une tempête pour préserver Alcmène de la mort par le feu.

Une mise en scène comique de la stratégie du bûcher est attestée sur la scène des Thesmophories d'Aristophane: Mnesilochos, poursuivi par les femmes qui sont en train de célébrer la fête, cherche refuge sur un autel. Les femmes menacent de mettre le feu, avec les flambeaux utilisés pour les Thesmophories, à l'autel présent sur scène, mais elles n'y arrivent pas: un prytanis intervient pour sauver Mnesilochos du bûcher ... et le condamner à mort par exposition.

Le bûcher a donc la fonction d'accélérer les temps du jugement sur le suppliant, dans la mesure où il oblige les autorités à intervenir en faveur de ou contre celui-ci. En même temps, la mise à feu de l'espace sacré pousse les dieux à intervenir pour sauver le suppliant ou au contraire pour décider de le laisser mourir.

\section{La flamme sur l'autel : entre action rituelle et représentation dramatique}

En conclusion, dans cet article nous avons observé les différentes configurations dramatiques d'une image rituelle, le feu sur l'autel. Ce motif est, d'une part, une façon alternative de représenter le sacrifice - en lieu et place de la $\sigma \varphi \propto \gamma \eta ́$ et du registre du rite corrompu - et, d'autre part, constitue une des dérives violentes et impies du rite de la supplication.

Dans plusieurs passages tragiques, l'image du feu du sacrifice sur les autels, avec ses différentes représentations, est utilisée par les poètes afin de mobiliser différents aspects des rites, avec diverses conséquences dans la construction de l'intrigue et dans la caractérisation des personnages. 


\section{BIBLIOGRAPHIE}

Ackermann D. (2007), « Rémunération des prêtres et déroulement des cultes dans le règlement religieux d'Aixônè (Attique) », Les Études Classiques 75, p. 111-136.

Amandry P. (1950), La mantique apollinienne à Delphes : essai sur le fonctionnement de l'Oracle, Paris.

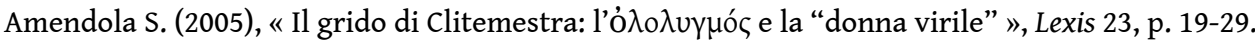

Beekes R. S. P. (2010), Etymological Dictionary of Greek, Leiden.

Bremmer J. N. et Veenstra J. R. (2002), The Metamorphosis of Magic from Late Antiquity to the Early Modern Period, Louvain.

Brouillet M. et Carastro C. éd. (2018), Dossier : Place aux objets! Présentification et vie des artefacts en Grèce ancienne, Mètis N.S.16, p. 7-165. 
Bruit Zaidman L. (2005), « Offrandes et nourritures : repas des dieux et repas des hommes en Grèce ancienne ", dans Georgoudi S., Koch-Piettre R. et Schmitt Pantel P. éd., La cuisine et l'autel : les sacrifices en questions dans les sociétés de la Méditerranée ancienne, Turnhout, p. 31-46.

Burkert W. (1966), « Greek Tragedy and Sacrificial Ritual », GRBS 7-2, p. 87-121.

- (1972), Homo necans : Interpretationen altgriechischer Opferriten und Mythen, Berlin-New York.

Calame C. (1997), « De la poésie chorale au stasimon tragique », Métis XII, p. 181-203.

- (1998), « Héraclès, animal et victime sacrificielle dans les Trachiniennes de Sophocle ? », dans Bonnet C., Jourdain-Annequin C. et Pirenne-Delforge V. éd., Le Bestiaire d'Héraclès. III ${ }^{e}$ Rencontre héracléenne, Kernos supplément 7, Liège, p. 197-215.

- (2013), « De la pratique culturelle dominante à la philologie classique : le rôle du chœur dans la tragédie attique ", LEXIS 31, p. 16-28.

- (2017), La tragédie chorale : poésie grecque et rituel musical, Paris.

Carastro C. (M.) (2006), La cité des mages : penser la magie en Grèce ancienne, Grenoble.

Casabona J. (1966), Recherches sur le vocabulaire des sacrifices en grec des origines à la fine de l'époque classique, Aix-en-Provence.

Chantraine P. (1968), Dictionnaire étymologique de la langue grecque : histoire des mots, Paris.

Collins D. (2008), Magic in the Ancient Greek World, Malden.

Decourt J.-C. et Tziaphalias A. (2015), « Un règlement religieux de la région de Larissa », Kernos 28, p. 13-51.

Denniston J. D. et Page D. L. (1957), Aeschylus, Agamemnon, Oxford.

Detienne M. et Vernant J.-P. (1979), La cuisine du sacrifice en pays grec, Paris.

Durand J.-L. (1986), Sacrifice et labour en Grèce ancienne : essai d'anthropologie religieuse, Paris-Rome.

Ekroth G. (2002), The Sacrificial Rituals of Greek Hero-Cults in the Archaic to the Early Hellenistic Periods, Liège.

- (2008), « Burnt, Cooked or Raw? Divine and Human Culinary Desires at Greek Animal Sacrifice ", dans Stavrianopoulou E., Michaels A. et Ambos C. éd., Transformations in Sacrificial Practices: from Antiquity to Modern Times. Proceedings of an International Colloquium, Heidelberg, 12-14, July 2006, Münster, p. 87-112.

- (2009), «Thighs or Tails? The Osteological Evidence as a Source for Greek Ritual Norms », dans Brulé P. éd., La norme en matière religieuse en Grèce ancienne. Actes du XII colloque international du CIERGA (Rennes, septembre 2007), Kernos supplément 21, Liège, p. 125-151.

- (2011), « Meat for the Gods », dans Pirenne-Delforge V. et Prescendi F. éd., " Nourrir les Dieux ? » Sacrifice et représentation du divin, Kernos supplément 26, Liège, p. 15-42.

- (2017), « Bare Bones: Zooarchaeology and Greek Sacrifice », dans Hitch S. et Rutherford I. éd., Animal Sacrifice in the Ancient Greek World, Cambridge, p. 15-47.

Fraenkel E. (1950), Aeschylus, Agamemnon, Oxford.

Friedrich R. (1998), « Everything to do with Dionysos? Ritualism, the Dionysiac, and the Tragic », dans Silk M. S. éd., Tragedy and the Tragic: Greek Theatre and beyond, Oxford, p. 257-283.

Furley W. D. (1981), Studies in the Use of Fire in ancient Greek Religion, New York.

Gell A. (1998), Art and Agency, Oxford. 
Georgoudi S. (2005), «L'“occultation de la violence” dans le sacrifice grec : données anciennes, discours modernes ", dans Georgoudi S., Koch Piettre R. et Schmidt F. éd., La cuisine et l'autel : les sacrifices en questions dans les sociétés de la Méditerranée ancienne, Turnhout, p. 115-147.

- (2016), « Des bêtes pleines à Athéna ? Un sacrifice pas si étrange », Pallas 100, p. 91-102.

Georgoudi S., Koch-Piettre R. et Schmidt F. (2005), La cuisine et l'autel : les sacrifices en questions dans les sociétés de la Méditerranée ancienne, Turnhout.

- (2011), La raison des signes : présages, rites, destin dans les sociétés de la Méditerranée ancienne, Leiden.

Gernet L. (1983), « You-you en marge d'Hérodote », dans Les Grecs sans miracle, textes réunis par R. Di Donato, Paris, p. 249.

Gill D. (1974), « “Trapezomata”: a Neglected Aspect of Greek Sacrifice », HThR 67-2, p. 117-137.

Giordano M. (1999), La supplica: rituale, istituzione sociale e tema epico in Omero, AION Sezione filologico-letteraria, Quaderni 3, Naples.

Goldhill S. (1984), Language, Sexuality, Narrative: The Oresteia, Cambridge.

Gould J. (1973), « Hiketeia », JHS 93, p. 74-103.

Graf F. (2006), « Drama and Ritual: Evolution and Convergences », dans Medda E., Mirto M. S. et Pattoni M. P. éd., Komoidotragoidia: intersezioni del tragico e del comico nel teatro del V secolo a.C., Pise, p. 103-118.

Henrichs A. (2000), « Drama and Dromena: Bloodshed, Violence, and Sacrificial Metaphor in Euripides », HSPh 100, p. 173-188.

- (2004), " "Let the Good Prevail": Perversions of the Ritual Process in Greek Tragedy ", dans Yatromanolakis D. et Panagiotis R. éd., Greek Ritual Poetics, Cambridge (Mass.), p. 189-198.

- (2012), « Animal Sacrifice in Greek Tragedy: Ritual, Metaphor, Problematizations », dans Faraone C. A. et Naiden F. S. éd., Greek and Roman Animal Sacrifice: Ancient Victims, Modern Observers, Cambridge, p. 180-194.

Houseman M. et Severi C. (2009), Naven ou le donner à voir. Essai d'interprétation de l'action rituelle, Paris.

Hubert H. et Mauss M. (1897), « Essai sur la nature et la fonction du sacrifice ", L'Année sociologique 2, p. 29-138.

Humphrey C. et Laidlaw J. (1994), The Archetypal Actions of Ritual: A Theory of Ritual. Illustrated by the Jain Rite of Worship, Oxford.

- (2006), « Action » dans Kreinath J., Snoek J. et Stausberg M. éd. Theorizing Rituals, Leiden, p. 265-284.

Jameson M. H. (1986), « Sophocles, Antigone, 1005-1022: an illustration », dans Cropp M., Conacher D. J., Fantham E. et Scully S. E. éd., Greek Tragedy and Its Legacy: Essays Presented to D.J. Conacher, Calgary-Alberta, p. 59-65.

Kearns E. (1994), « Cakes in Greek sacrifice regulations », dans Hägg R. éd., Ancient Greek Cult Practice from the Epigraphical Evidence. Proceedings of the Second International Seminar on Ancient Greek Cult Organized by the Swedish Institute of Athens, 22-24 November 1991, Stockholm, p. 65-70.

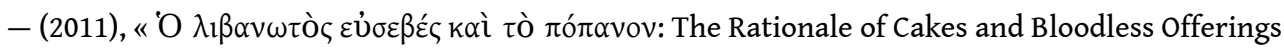
in Greek Sacrifice », dans Pirenne-Delforge V. et Prescendi F. éd., " Nourrir les Dieux ? » Sacrifice et représentation du divin, Kernos supplément 26, Liège, p. 89-104. 
Loraux N. (1985), Façons tragiques de tuer une femme, Paris.

Marrucci L. (2004), « Il buono (e il cattivo) uso della makhaira », LEXIS 22, p. 397-414.

Medda E. (2004), « Clitemestra e il Coro. Riflessioni sceniche e testuali su Aesch. Ag. 489-502 », LEXIS 22, p. 61-86.

- (2017), Eschilo, Agamennone, Rome.

Mehl V. et Brulé P. (2008), Le sacrifice antique : vestiges, procédures et stratégies, Rennes.

Mugelli G. (2018), « Eracle e il sacrificio interrotto: immagini tragiche di sacrificio nelle Trachinie di Sofocle e nell'Eracle di Euripide », Scienze dell'Antichità 23-3, p. 123-139.

Naiden F. S. (2006), Ancient Supplication, Oxford.

- (2013), Smoke Signals for the Gods: Ancient Greek Sacrifice from the Archaic through Roman Periods, Oxford.

Neils J. et Barber E. J. W. (1992), Goddess and Polis: the Panathenaic Festival in ancient Athens, Hanovre.

Parisinou E. (2000), The Light of the Gods The Role of Light in Archaic and Classical Greek Culture, Bristol.

Parker R. (2005), Polytheism and Society at Athens, Oxford.

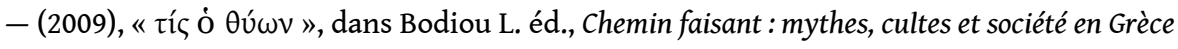
ancienne. Mélanges en l'honneur de Pierre Brulé, Rennes, p. 167-171.

- (2010), « New problems in Athenian religion: the "sacred law" from Aixone ", dans Bremmer J. N., Dijkstra J., Kroesen J. E. A. et Kuiper Y. éd., Myths, Martyrs and Modernity: Studies in the History of Religions in Honour of Jan N. Bremmer, p. 193-208.

- (2011), On Greek Religion, Londres-Ithaca.

Parker R. et Scullion S. (2016), « The Mysteries of the Goddess of Marmarini », Kernos 29, p. 209-266.

Pedrina M. (2017), La supplication sur les vases grecs : mythes et images, Pise.

Pritchett W. K. (1974), The Greek State at War, Berkeley.

Pulleyn S. (1997), Prayer in Greek Religion, Oxford.

Rodighiero A. (2004), Sofocle, La morte di Eracle (Trachinie), Venise.

Scullion S. (1994), « Olympian and Chtonian », ClAnt 13-1, p. 75-119.

- (2000), « Heroic and Chthonian Sacrifice: New Evidence from Selinous », ZPE 132, p. 163-171.

- (2009), « Sacrificial Norms, Greek and Semitic: Holocausts and Hides in a Sacred Law of Aixone ", dans Brulé P. éd., La norme en matière religieuse en Grèce ancienne. Actes du XIIe colloque international du CIERGA (Rennes, septembre 2007), Kernos supplément 21, Liège, p. 153-169.

Seaford R. (1998), « Something to Do with Dionysos - Tragedy and the Dionysiac: Response to Friedrich ", dans Silk M. S. éd., Tragedy and the Tragic: Greek Theatre and Beyond, Oxford, p. 284-309.

Severi C. (2017), L'objet-personne : une anthropologie de la croyance visuelle, Paris.

Sourvinou-Inwood C. (2003), Tragedy and Athenian Religion, Lanham.

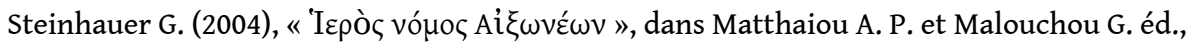

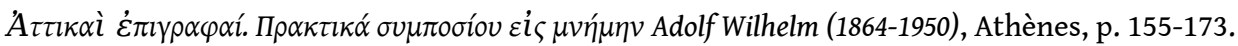


Svenbro J. (2005), « La thusia et le partage. Remarques sur la "destruction" par le feu dans le sacrifice grec », dans Georgoudi S., Koch Piettre R. et Schmidt F. éd., La cuisine et l'autel : les sacrifices en questions dans les sociétés de la Méditerranée ancienne, Turnhout, p. 217-225.

Taddei A. (2009), « Inno e pratiche rituali in Euripide: il caso dell'Ifigenia tra i Tauri », Paideia LXIV, p. 235-252.

- (2014), « Le Panatenee nel terzo stasimo degli Eraclidi (Eur. Heracl. 748-783) », LEXIS 32, p. 213-228.

- (2015), « Ifigenia e il Coro nella "Ifigenia tra i Tauri”. Destini rituali incrociati », LEXIS 33, p. $150-167$.

- (2016), «Vergognarsi davanti al proprio dio: il coro nel terzo stasimo dello Ione di Euripide », Quaderni Urbinati di Cultura Classica 113-2, p. 47-64.

Taplin O. (1977), The Stagecraft of Aeschylus: the Dramatic Use of Exits and Entrances in Greek Tragedy, Oxford.

- (2007), Pots \& Plays: Interactions Between Tragedy and Greek Vase-painting of the Fourth Century B.C., Los Angeles.

Van Straten F. T. (1988), « The God's Portion in Greek Sacrificial Representations: Is The Tail Doing Nicely? », dans Hägg R., Marinatos N. et Nordquist G. éd., Early Greek Cult Practice. Proceedings of the Fifth International Symposium at the Swedish Institute at Athens, 26-29, June, 1986, Stockholm, p. 51-68.

- (1995), Hierà Kalà: Images of Animal Sacrifice in Archaic and Classical Greece, Leiden.

Vernant J.-P. (1981), « Théorie générale du sacrifice et mise à mort dans la Өvoí $\alpha$ grecque », dans Rudhardt J. éd., Le sacrifice dans l'Antiquité, Genève, p. 1-21.

Vidal-Naquet P. (1972), « Chasse et sacrifice dans l'Orestie d'Eschyle », dans Vernant J.-P. et VidalNaquet P., Mythe et tragédie en Grèce ancienne, Paris, p. 133-158.

Winkler J. J. et Zeitlin F. I. (1990), Nothing to Do with Dionysos: Athenian Drama in Its Social Context, Princeton.

Zeitlin F. I. (1965), « The Motif of the Corrupted Sacrifice in Aeschylus'Oresteia », TAPhA 96, p. 463-508.

\section{NOTES}

1. Chantraine 1968, s.v. Өúw, voir aussi Casabona 1966, p. 69-154. Pour le verbe $\theta u ́ \omega$ et le nom $\theta v \sigma i ́ \alpha$, les interprètes ont identifié un usage «technique " qui indique le sacrifice sanglant suivi par un banquet, opposé aux sacrifices "héroïques » sous la forme de la

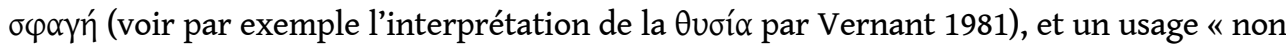
marqué » qui indique génériquement un sacrifice. À propos des emplois de $\theta u ́ \omega$ et $\theta v \sigma i ́ \alpha$ par rapport aux pratiques sacrificielles réelles voir Scullion 1994, Ekroth 2002, Parker 2011, p. 124-170.

2. Les différents types d'offrandes non sanglantes, surtout sous la forme de gâteaux, pouvaient être brulés sur l'autel ou déposés sur la table sacrificielle, voir Kearns 1994, Bruit Zaidman 2005 et Kearns 2011. À propos des trapezomata voir Gill 1974. Sur l'usage du feu dans la religion grecque voir Furley 1981. 
3. La répartition de la viande sacrificielle entre hommes et dieux a été interprétée par Detienne et Vernant 1979. À propos de la part des dieux dans l'iconographie voir Van Straten 1988 et 1995, p.115-160. Les résultats les plus intéressants à propos de la répartition de la viande ont été atteints par les travaux de G. Ekroth 2008, 2009, 2011 et 2017. Dans Ekroth 2002, l'auteure s'est interrogée sur la question des pratiques sacrificielles telles que l'holocauste et l'enagismos, démarches sacrificielles où le feu était évidemment fondamental. À propos des pratiques de partage de la viande sacrificielle, voir aussi la notion de «moirocaust » par Scullion 2000. J. Svenbro (2005) développe des considérations intéressantes à propos du concept de consommation / destruction par le feu et le partage dans la $\theta u \sigma i ́ \alpha$. Enfin, une analyse du processus sacrificiel en fonction de la communication entre les hommes et les dieux, qui pouvaient accepter ou refuser le sacrifice, est développée par Naiden 2013.

4. La notion de "perverted sacrifice» a été introduite par F. Zeitlin (1965) pour interpréter les métaphores sacrificielles dans l'Orestie et développée par P. Vidal-Naquet

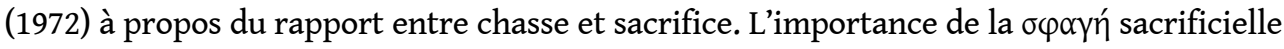
dans la tragédie grecque a été soulignée par Burkert 1966, qui a mis l'accent sur l'égorgement dans sa théorie générale du sacrifice grec (Burkert 1972). S. Georgoudi (2005) a bien montré que le problème de l'égorgement et de la violence sacrificielle n'était pas au centre de la réflexion des anciens à propos de la pratique du sacrifice. À propos de la catégorie du « perverted ritual » et de son applicabilité à la tragédie grecque voir les travaux de Henrichs 2000, 2004 et 2012.

5. J'ai abordé la question des différents pattern de repésentation du rite à propos des sacrifices d'Héraclès chez Sophocle et Euripide dans Mugelli 2018.

6. À propos du rapport entre tragédie et religion grecque voir Sourvinou-Inwood 2003. F. Graf (2006) a résumé les différentes théories à propos du rapport entre rituel et tragédie. À propos de la compétence rituelle des spectateurs voir les travaux de Taddei 2009, 2014, 2015 et 2016.

7. À propos des objets qui agissent en tant que social agents, voir Gell 1998.

8. Voir Severi 2017 à propos de l'anthropomorphisme des objets.

9. La théorie de l'action rituelle proposée par Humphrey et Laidlaw 1994, attribue un rôle déterminant, dans le processus de ritualisation des actions "ordinaires", à l'intentionnalité des agents rituels. Voir également Humphrey et Laidlaw 2006, pour un examen des différentes perspectives théoriques sur le rituel en tant que mode d'action. En particulier, les auteurs prennent en compte la perspective relationnelle adoptée par Houseman et Severi 2009.

10. À propos des différentes stratégies de "présentification » des artefacts voir le dossier de Métis N.S.16, 2018, et notamment l'introduction par M. Brouillet et C. Carastro.

11. Euripide, Iphigénie à Aulis, 1470-1472. La flamme du sacrifice est mentionnée en association avec les graines et la corbeille utilisées dans les actions préliminaires au sacrifice.

12. Le fragment plus ancien de l'inscription est contenu dans IG II $^{2} 1356=$ LSCG $28=$ SEG 54,214, 400-375 a. C. Pour l'édition complète voir Steinhauer 2004 et CGRN 57 (http:// cgrn.ulg.ac.be/file/57). À propos des différentes pratiques sacrificielles prévues dans l'inscription et la distribution de la viande sacrificielle, voir Ackermann 2007, Scullion 2009, Parker 2010. La prescription de bois additionnel pour les holocaustes est attestée 
dans l'inscription de Marmarini, publiée par Decourt et Tziaphalias 2015; voir le commentaire par Parker et Scullion 2016, p. 252-253.

13. LSS 19 = SEG 21, 257 = CGRN 84 (http://cgrn.ulg.ac.be/file/84), 363/2 a.C., voir Georgoudi 2016.

14. LSS 22 = SEG 45, 272 = CGRN 64 (http://cgrn.ulg.ac.be/file/64).

15. Euripide, Électre, 800-802.

16. La phase préliminaire à la mise à mort, dans laquelle les instruments du sacrifice circulent près de l'autel, est la plus représentée dans l'iconographie du sacrifice, voir Van Straten 1995, p. 186-192.

17. Voir par exemple Sophocle, Philoctète, 1032-1033.

18. Dans Euripide, Oreste, 1137, Pylade imagine la réaction joyeuse de tous les Grecs à la

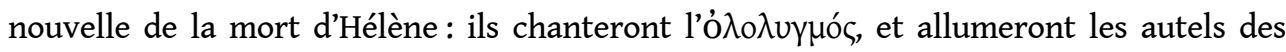
dieux avec le feu du sacrifice.

19. S. Amendola (2005) a discuté de l'emploi de ce type de cri rituel dans l'Agamemnon. À

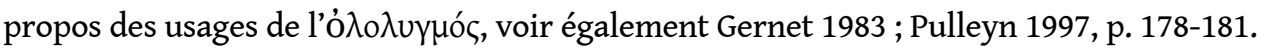

20. Il s'agit du modèle des rites qui suivent une $\varepsilon \dot{u} \alpha \gamma \gamma \varepsilon \lambda i ́ \alpha$. Ces sacrifices peuvent être associés à d'autres types de sacrifices post eventum d'action de grâce, tels que les

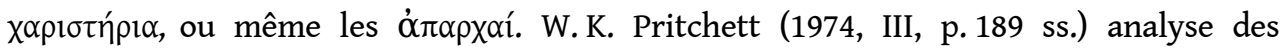
exemples de ces rites en contexte militaire, qui toutefois ne sont pas contemporains de l'Athènes d'Eschyle) : à l'occasion de la mort de Philippe de Macédoine, par exemple, la ßou入ń des Athéniens avait organisé des sacrifices. À propos du rapport entre l'action rituelle, les événements qui la précèdent et les intentions des sacrifiants, le cas d'Agésilas est très intéressant : lors de l'annonce de la défaite de l'armée, Agésilas accomplit de faux sacrifices pour la bonne nouvelle, afin de ne pas décourager son armée (Xénophon, Helléniques IV, 3, 14).

21. Eschyle, Agamemnon, 88-96. Voir le texte et le commentaire par Medda 2018.

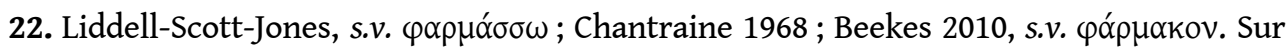
l'ambiguité du champ sémantique de la $\varphi \alpha \rho \mu \alpha \kappa \varepsilon i ́ \alpha$, entre médecine, religion et magie, cf. Bremmer et Veenstra 2002, p. 1-11 ; Parker 2005, p. 116-135 ; Carastro 2006, p. 141-159 ; Collins 2008, p.133-135. Sur les ambiguïtés lexicales contenues dans ce passage, cf. Goldhill 1984, p. 7-18 ; Medda 2017, p. 94-95.

23. Neils et Barber 1992 ; Parisinou 2000 ; Parker 2005, p. 257-258.

24. Le $\pi \varepsilon \lambda \alpha v o ́ \varsigma$ pouvait être payé en argent, voir Amandry 1950, p. 86-103. L'offrande du $\pi \varepsilon \lambda \alpha v o ́ \varsigma$ à Éleusis était contrôlée par les Eumolpides (IG I ${ }^{3}$ 1-2, 422 a.C. ; IG II ${ }^{2}$ 140, 353-352 a.C.).

25. Si on exclut les inscriptions, à l'âge classique le mot $\pi \varepsilon \lambda \alpha v o ́ \varsigma$ n'est attesté que dans des textes tragiques.

26. À propos de l'agentivité rituelle du chœur dans cette tragédie, voir Taddei 2016.

27. Eschyle, Agamemnon, 587. À propos de la mise en scène de ce passage voir Medda 2004. O. Taplin (1977) imagine que Clytemnestre n'est pas présente sur scène au moment de l'annonce du héraut.

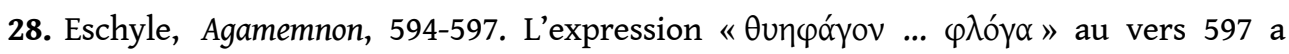
inspiré le titre de cet article. L'image du feu en train de s'éteindre a été associée par Fraenkel 1950 aux libations de vin, qui dans l'Iliade et l'Odyssée sont versées en conclusion 
du sacrifice. J. D. Denniston et D. L. Page 1957 parlent d'encens jeté sur la flamme, qui transforme le feu en une flamme parfumée. À propos des problèmes de genre liés à

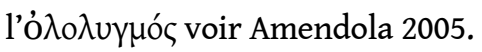

29. Jameson 1986 ; Ekroth 2009.

30. Le geste a été reconstruit par Jameson 1986 qui a mis en rapport le passage de l' Antigone de Sophocle (1005-1022) avec l'image du cratère à figure rouge peinte par Cléophon (Hermitage ГР-4592, moitié $\mathrm{V}^{\mathrm{e}}$ siècle a.C.)

31. D'après Xénophon, par exemple, un commandant devait apprendre à lire les entrailles, pour éviter les tricheries des devins (Xénophon, Cyropédie, I, 6, 2 ; Memorabilia, I, 1, 6-9 et l'exemple d'Agésilas dans les Helléniques, III, 4, 15).

32. Pausanias V, 14, 1. Voir Naiden 2013, p. 138.

33. Sur la supplication en général, voir Gould 1973 et Giordano 1999. F. S. Naiden (2006) a discuté, en particulier, les dynamiques entre requête du suppliant et réponses du supplié.

34. Euripide, Andromaque, 257-260.

35. Le choix de la $\sigma \varphi \alpha \gamma \eta ́$, mort virile par excellence, est fait par Mégara qui critique l'obstination d'Amphitryon; à propos des mises à mort masculines et féminines dans la tragédie voir Loraux 1985.

36. Naiden 2006, p. 154.

37. Chez Hérodote VI, 78-84, Cléomène de Sparte est convaincu par un oracle trompeur d'Apollon d'attaquer les Argiens, qui ont cherché refuge dans un bois sacré. Cléomène essaye dans un premier moment de faire sortir les suppliants du lieu sacré, mais après plusieurs tentatives, sûr de la faveur d'Apollon, il met le feu au bois et tue les suppliants. À propos de la mort de Cléomène, qui est représentée comme un usage perverti du couteau sacrificiel, voir Marrucci 2004.

38. Dans l'épisode, raconté par Diodore de Sicile, XVI, 58, de la mise à mort des Phocéens pendant la troisième guerre sacrée, c'est Apollon lui-même qui décide de condamner les Phocéens et met le feu aux stibades et aux tentes qui se trouvent dans son sanctuaire. Selon Pausanias X, 35, 3, les Phocéens sont en train de supplier dans le sanctuaire de Déméter, mis à feu par les Thébains.

39. Voir aussi le processus d'attribution de responsabilité dans la mise à mort par le bûcher dans l'épisode de la mort d'Héraclès chez Sophocle, Trachiniennes, 1210-1215 : Lycos accepte de prendre en charge les funérailles de son père encore vivant et de construire le bûcher, mais pas d'allumer le feu avec sa torche, en refusant de cette façon d'avoir un rôle actif dans la mise à mort du héros; voir Calame 1997. L'allumage du bûcher est pris en charge par Philoctète, ou par son père, voir Rodighiero 2004, p. 1214-1215.

40. Voir Taplin 2007, p. 170-174. À propos de la série iconographique d'Alcmène sur le bûcher voir Pedrina 2017, p. 89-132. 


\section{RÉSUMÉS}

Compte tenu de l'immense variété des pratiques sacrificielles grecques, la présence de la flamme sur l'autel était l'un des rares éléments constants dans l'expérience de tout sacrifiant. Dans la consécration des offrandes sanglantes et non sanglantes, la présence d'un feu vif sur l'autel était indispensable à la bonne réussite du rite. Dans la tragédie, où le sacrifice est souvent représenté en tant que "rituel corrompu», le feu est une stratégie de mise en image du sacrifice, qui se présente comme une alternative à la description de la violence sacrificielle, centrée sur le sang. En observant les croisements entre l'expérience du rite et l'expérience de la tragédie, cet article analyse les différentes configurations dramatiques de l'image du feu qui brûle sur l'autel. Cette image rituelle polyvalente est employée par les poètes tragiques afin de mobiliser les différentes dynamiques de l'action sacrificielle. Les modes d'actions tragiques du feu sacrificiel sont donc explorés dans l'interaction avec les espaces et les agents du sacrifice, avec le but d'observer les réseaux d'actions qui déterminent l'efficacité du rite. Observer comment une image rituelle s'adapte à l'intrigue de la tragédie nous permet de réfléchir à la tragédie en tant qu'expérience rituelle complexe, qui exploite les différentes formes de la plasticité du rite.

Given the enormous variety of Greek sacrificial practices, the presence of fire on the altar was to be one of the few constant elements in the experience of the participants : in blood and bloodless offerings, the presence of a bright fire was essential for the good outcome of the rite. In tragedy, where the sacrifice is often portrayed as a perverted ritual, the description of the sacrificial flame is an alternative strategy to represent the sacrifice, instead of focusing on the blood of the victim, the slaughter and the sacrificial violence. Focusing on the interferences between the ritual and the dramatic experience, this article analyses the different dramatic configurations of a ritual image in the texts of Greek tragedy. The image of the fire burning in the sacred space is used by the tragic poets to represent the different dynamics of the sacrificial action. The tragic modes of action of the sacrificial fire are thus explored in the interaction with the ritual spaces and the agents of the sacrifice, focusing on the networks of actions which determine the effectiveness of the rite. Observing how a ritual image is adapted to the plot of the tragedy allows us to reflect on the tragedy as a complex ritual experience, which exploits the various forms of plasticity of the rite.

\section{INDEX}

Keywords : fire, sacrifice, agency, tragedy, ritual practice, performance, supplication, ritual Mots-clés : feu, sacrifice, agentivité, tragédie, pratique rituelle, performance, rituel, supplication

\section{AUTEUR}

\section{GLORIA MUGELLI}

Docteure de l'Université de Pise et de l'EHESS 